\title{
Pax3 regulation of FGF signaling affects the progression of embryonic progenitor cells into the myogenic program
}

\author{
Mounia Lagha, ${ }^{1}$ Jay D. Kormish, ${ }^{1,3}$ Didier Rocancourt, Marie Manceau, ${ }^{4}$ Jonathan A. Epstein, ${ }^{5}$ \\ Kenneth S. Zaret, ${ }^{3}$ Frédéric Relaix ${ }^{2}{ }^{2}$ and Margaret E. Buckingham ${ }^{6}$
}

CNRS URA 2578, Department of Developmental Biology, Pasteur Institute, 75015 Paris, France

Pax3/7-dependent stem cells play an essential role in skeletal muscle development. We now show that Fgfr4 lies genetically downstream from $\mathrm{Pax} 3$ and is a direct target. In chromatin immunoprecipitation (ChIP)-on-chip experiments, Pax3 binds to a sequence 3' of the Fgfr4 gene that directs Pax3-dependent expression at sites of myogenesis in transgenic mouse embryos. The activity of this regulatory element is also partially dependent on E-boxes, targets of the myogenic regulatory factors, which are expressed as progenitor cells enter the myogenic program. Other FGF signaling components, notably Sprouty1, are also regulated by Pax3. In vivo manipulation of Sprouty expression reveals that FGF signaling affects the balance between Pax-positive progenitor cells and committed myoblasts. These results provide new insight into the Pax-initiated regulatory network that modulates stem cell maintenance versus tissue differentiation.

[Keywords: Pax3; Fgfr4; Sprouty1; myogenesis; embryonic ChIP-on-chip; somite; skeletal muscle progenitor cells]

Supplemental material is available at http://www.genesdev.org.

Received February 28, 2008; revised version accepted May 9, 2008.

The balance between stem cell self-renewal and progression into a differentiation program is of critical importance for tissue growth and regeneration. During skeletal muscle development, each muscle contains a pool of resident muscle stem cells that can either differentiate into muscle fibers or remain as proliferating progenitors. These cells express two related paired-homeobox transcription factors, Pax3 and Pax7, that are essential for ensuring the myogenic potential and survival of the progenitors (Buckingham and Relaix 2007). In the embryo, skeletal muscle is derived from transitory segmented structures called somites. Pax3/Pax7-positive muscle progenitor cells are located in the dermomyotome, which constitutes the dorsal epithelial layer of each somite. These cells enter the myotome, the first skeletal muscle to form, in the central compartment of the somite, as the dermomyotome disaggregates, from embry-

\footnotetext{
${ }^{1}$ These authors contributed equally to this work.

Present addresses: ${ }^{2}$ UMR S 787, INSERM-UPMC-Paris VI, Faculté de Médecine Pitié-Salpétrière, 75634 Paris, France; ${ }^{3}$ Cell and Developmental Biology Program, Fox Chase Cancer Center, 333 Cottman Avenue, Philadelphia, PA 19111, USA; ${ }^{4}$ IBDM, Université de la Méditerranée, CNRS, UMR 6216, Campus de Luminy, Case 907, 13288 Marseille, France; ${ }^{5}$ Department of Cell and Developmental Biology, University of Pennsylvania, 1154 BRB II, 421 Curie Boulevard, Philadelphia, PA 19104, USA.

${ }^{6}$ Corresponding author.

E-MAIL margab@pasteur.fr; FAX 331-40-613452.

Article is online at http://www.genesdev.org/cgi/doi/10.1101/gad.477908.
}

onic day 10.5 (E10.5) in the mouse embryo (Relaix et al. 2005). Pax3 is more extensively expressed than Pax7, initially, and Pax3 mutant embryos display somite defects with loss of the epaxial and hypaxial extremities of the dermomyotome. Muscles such as those of the limb, which normally form after delamination and migration of muscle progenitor cells from the hypaxial dermomyotome, are absent in the Pax3 mutant. Progenitor cell delamination and migration depends on $c$-Met (Bladt et al. 1995), which is a Pax3 target (Epstein et al. 1996; Relaix et al. 2003). As they enter myogenesis, muscle progenitor cells down-regulate Pax3 and Pax7 and activate genes for the myogenic determination factors Myf5, Mrf4, and MyoD. Subsequent muscle differentiation depends on MyoD, Mrf4, or myogenin (Buckingham 2006). During development, each forming muscle mass therefore contains a heterogeneous population of cells reflecting the multiple steps of myogenesis. The decision to enter the myogenic program or to stay undifferentiated is a crucial choice that remains poorly understood. One way to approach this process is to identify relevant Pax3 target genes. Recently it was shown that the myogenic determination gene Myf5 is a direct Pax3 target (Bajard et al. 2006). However, entry into the myogenic program and skeletal muscle differentiation must be modulated to retain the progenitor cell pool.

Signaling pathways have been largely implicated in stem cell behavior. The FGF signaling pathway has been 
reported to act on stem cell fate in different developing organs such as liver (Calmont et al. 2006), the auditory sensory epithelium (Shim et al. 2005), or the tooth (Klein et al. 2008). FGF signaling has been implicated in myogenesis in vivo, both in promoting progenitor cell proliferation (von Scheven et al. 2006) and in promoting their differentiation (Buckingham 2006; Hammond et al. 2007). In the embryo, Fgfr4 is transcribed at sites of myogenesis (Stark et al. 1991), in replicating myoblasts, but is not detected in avian muscle fibers (Marcelle et al. 1995). More recently, colocalization of Fgfr4 transcripts with Pax7 in muscle progenitor cells has been reported in the chick embryo (Ben-Yair and Kalcheim 2005). Misexpression of Fgf4 in the chick limb inhibits myogenesis by down-regulating Fgfr4 (Edom-Vovard et al. 2001), and indeed electroporation of a dominant-negative form of Fgfr4 prevents expression of the myogenic regulatory factors, whereas Pax3 remains unchanged (Marics et al. 2002). Despite the evidence in the chick embryo that Fgfr4 is important for myogenesis, Fgfr4-null mice develop normally (Weinstein et al. 1998), which may reflect overlapping function with other FGF receptors.

In this study, we explore FGF signaling in the myogenic context in the mouse embryo. We establish that Fgfr4 lies genetically downstream from Pax3 at sites of myogenesis and show by chromatin immunoprecipitation (ChIP)-on-chip experiments that Fgfr4 is directly activated by Pax3 in vivo through a 3 ' regulatory element. This Pax3-dependent element also contains E-boxes, al- lowing subsequent regulation by myogenic determination factors, such as MyoD, in cells that have entered the myogenic program. We also show that other components of the FGF signaling pathway, notably Sprouty1, are regulated by Pax3 during myogenesis. These observations, together with in vivo manipulation of Sprouty, leads us to propose that Pax3, through regulation of FGF signaling, modulates muscle progenitor cell progression into the myogenic program.

\section{Results}

Fgfr4 lies genetically downstream from Pax3 during myogenesis

We examined Fgfr4 expression at different stages of development from E9.5 to E12.5 in different Pax3 genetic backgrounds (Fig. 1). In normal mouse embryos, Fgfr4 transcripts are detected at sites of myogenesis, notably in the somites at E9.5-E10.5 (Fig. 1A,C) and at later stages also in the limb buds and some head muscles (Fig. 1E,G).

In the presence of PAX3-FKHR, in which the DNAbinding domain of Pax3 is fused to the strong transcriptional activation domain of FKHR (FOXO1A) (Relaix et al. 2003), Fgfr4 is overexpressed in the somites at E9.5E10.5 (Fig. 1B,D). Conversely, in embryos that express a dominant-negative form of Pax3, Pax3-En, in which the DNA-binding domain of $\mathrm{Pax} 3$ is fused to the repression domain of Engrailed (Bajard et al. 2006), Fgfr4 transcripts
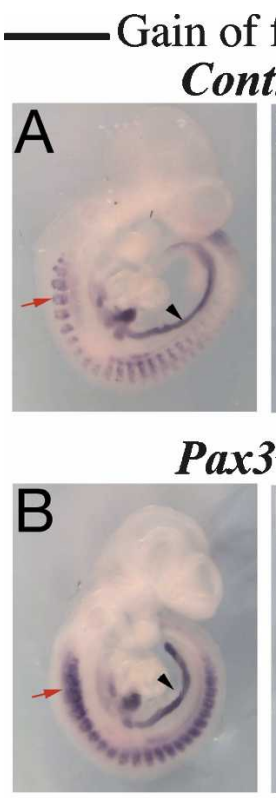

E9.5

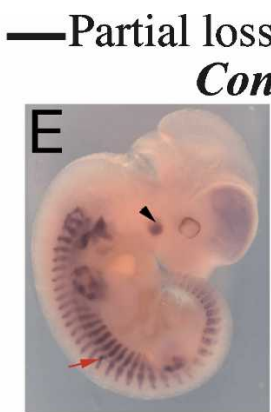
Control

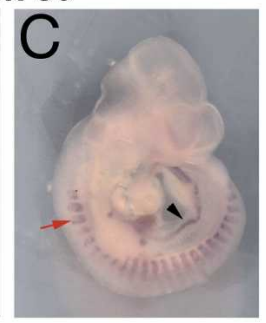

Pax3 $3^{\text {PAX3-FKHR/+ }}$

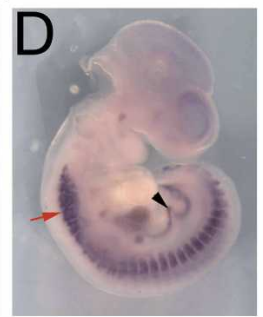

E10.5

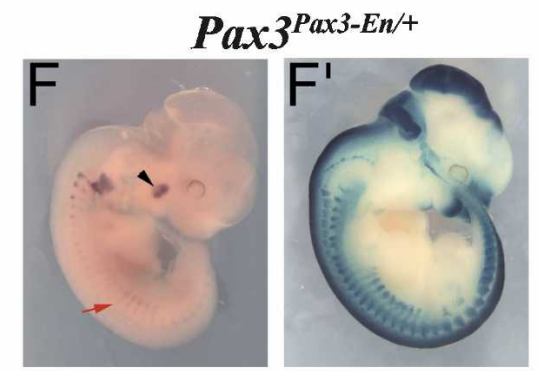

E11.5

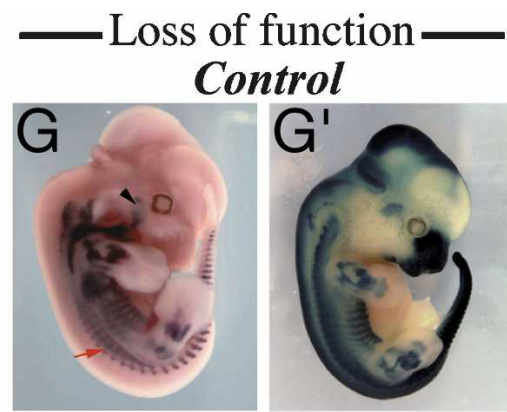

Pax3 $3^{\text {nlacZ/nlacZ }}$

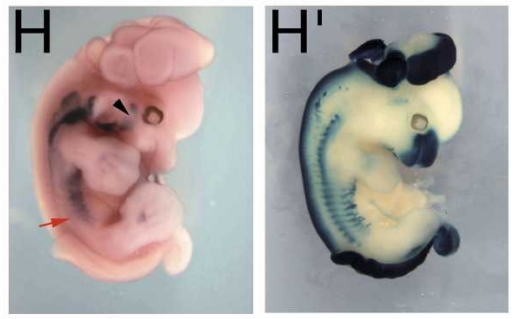

E12.5

Figure 1. Fgfr4 lies genetically downstream from $\operatorname{Pax} 3$ during myogenesis. $(A-H)$ Whole-mount in situ hybridization with a $F g f r 4$ probe, at the embryonic day (E) indicated. $(A, C, E, G)$ Control embryos. $(B, D)$ Pax $3^{\text {PAX3-FKHR-IRESnlacZ } /+}\left(\right.$ Pax $3^{\text {PAX3-FKHR/+ }}$ ) embryos expressing the gain-of-function allele of $\operatorname{Pax} 3 .(F)$ A $P a x 3^{\text {Pax3-En-IRESnlacZ } /+}\left(\operatorname{Pax}^{\text {Pax3-En } /+}\right)$ embryo expressing a dominant-negative allele of $P a x 3$ that in the heterozygote results in partially compromised $P a x 3$ function. $(H) P a x 3^{\text {nlacz/nlacz }}$, a null mutant of $P a x 3$. (E'- $\left.H^{\prime}\right)$ X-Gal staining of control $\left(E^{\prime}, G^{\prime}\right), \operatorname{Pax}^{\text {Pax3-En-IRESnlacZ/+ }}\left(F^{\prime}\right)$, and $\operatorname{Pax}^{\text {nlacZ/nlacZ }}\left(H^{\prime}\right)$ embryos to show the presence of Pax3-positive cells at the stages indicated. Red arrows point to differences in Fgfr4 transcripts at sites of myogenesis in the somites. Black arrowheads indicate internal controls at sites where Fgfr4 expression is not affected and Pax3 is not expressed. 
are down-regulated in somites and limb buds as shown at E11.5 (Fig. 1F). In Pax3 mutants, Fgfr4 expression is only detectable at some sites, notably in anterior muscles (Fig. $1 \mathrm{H}$ ) and at sites in the trunk where Pax7 is also directing myogenesis at E12.5 (Relaix et al. 2004, 2005). When Pax 3 function is impaired, apoptosis occurs. The presence of an nlacZ reporter in the Pax3 $3^{\text {Pax3-En-IRESnlacZ }}$ $\left(\operatorname{Pax}^{\text {Pax3-En }}\right)$ allele $\left(\right.$ Fig. $\left.1 \mathrm{~F}^{\prime}\right)$ and in the Pax3 $3^{\text {nlacZ/nlacZ }}$ mutant (Fig. $\left.1 \mathrm{H}^{\prime}\right)$ indicates the extent to which cells are lost, notably in the hypaxial extremity of the somites and limb buds. Nevertheless, labeled cells are present in $\mathrm{Pax}^{\mathrm{Pax} 3-\mathrm{En} /+}$ embryos, where Fgfr 4 transcripts are barely detectable (Fig. 1F). This effect is more severe than in the Pax3 mutant (Fig. 1H), probably because Pax3-En interferes with Pax7 as well as Pax3 activity, preventing Fgfr4 activation by either Pax factor Fgfr4 is not expressed at other non-myogenic sites of $\mathrm{Pax} 3$ expression, such as the neural tube (Fig. 1E, $E^{\prime}, G, G^{\prime}$ ). We therefore conclude that expression of Fgfr4 in the myogenic lineage depends on Pax3.

\section{Identification of a distal element in the Fgfr4 locus that binds Pax3 in vivo}

To test if Pax3 directly regulates Fgfr4 transcription, we performed an in vivo ChIP-on-chip experiment. To develop conditions for this assay, we used a transgenic line (P34) where five Pax3 consensus binding sites are placed upstream of a tk-nlacZ transgene (Relaix et al. 2004), which provides a readout of $\mathrm{Pax} 3 / \mathrm{Pax} 7$ transcriptional activity and therefore a positive control of Pax3/Pax7 binding to DNA. Somites and limb buds were pooled from P34 transgenic embryos at E11.5 (Fig. 2A). ChIP with Pax3 antibodies showed robust binding of Pax3 to the P34 element (Fig. 2B). We also detected Pax3 binding to a $-57.5-\mathrm{kb}$ element of the Myf5 gene that we had previously shown to be directly bound by Pax3 (Bajard et al. 2006). These results demonstrate that our ChIP protocol is efficient with small quantities of material.

The amplified ChIP product was hybridized to a custom-made NimbleGen array that probes sequences from genomic loci related to signaling and development. In the Fgfr4 tiled region, a domain, framed in Figure 2C, gave a strong hybridization signal in both biological replicates. This domain is $19.2 \mathrm{~kb}$ downstream from the Fgfr4 translation start site and $6.5 \mathrm{~kb} 3^{\prime}$ of the end of the gene. It gave enrichment with the original ChIP material, which was less than that seen for the -57.5-kb Myf5 element, but greater than that with Myf5 or Albumin negative controls (Fig. 2B).

The 50-mer with the highest hybridization signal in the embryonic Pax3 ChIP-on-chip data was positioned between 19,198 and 19,247 bp 3' of the gene. This lies within a 559-bp sequence that is highly conserved among mammals, present at 17,091, 15,919, and 12,039 bp downstream from the rat, human, and bovine Fgfr4 translation start codons, respectively (Fig. 2D). This sequence contains several potential Pax3-binding sites (Epstein et al. 1996; Bajard et al. 2006), indicated as P1-P6 in Figure 2D. Sites P4 and P5 are present in the $+19.2-\mathrm{kb}$ domain used in qPCR verification of the ChIP experiment (Fig. 2B). Gel mobility shift assays with an oligonucleotide containing these two Pax3-binding sites (Fgfr4-P4/P5) shows that it binds Pax3. Competition experiments indicate that this is mainly due to the $\mathrm{P} 4$ site (binding to an oligonucleotide from the consensus P34 transgene provides a positive control) (Fig. 2E). These experiments reveal a novel Pax3-binding region in the 3'-flanking sequence of the Fgfr4 gene.

\section{In vivo activity of the Fgfr4 distal element}

In order to test whether the 559-bp element will direct transcription to sites of myogenesis in the mouse embryo, it was inserted into a $t k-n l a c Z$ transgenic reporter (Hadchouel et al. 2000). In transgenic mouse embryos, $\mathrm{X}$-Gal staining showed $\beta$-galactosidase ( $\beta$-Gal) activity in the myotome and limb buds (Fig. 3C,D), resembling the endogenous Fgfr4 expression, shown at E11.5 and E12.5 (Fig. 3A,B). These results indicate that the 559-bp element is sufficient to direct myogenic expression of Fgfr4 in the embryo. We therefore investigated whether this is Pax3-dependent. When all six Pax sites were mutated, the transgene was no longer expressed at sites of Fgfr4 transcription (Fig. 3E,F), demonstrating that they are essential for the myogenic activity of the Fgfr 4 distal element. From these results, we conclude that myogenic expression of $\mathrm{Fgfr} 4$ is directly regulated by $\mathrm{Pax} 3 / \mathrm{Pax} 7$ acting through the 559-bp element.

We then looked more closely at expression in the somite and limbs. Fgfr4 is transcribed in the myotome (Supplemental Fig. S1), not in the dermomyotome, except for a weak expression in the extremities (data not shown), where Pax3 is also expressed. Immunohistochemistry experiments show colocalization of nuclear Pax3 staining (red) in cells that have surrounding Fgfr4 staining (green) (Fig. 3G,H). Pax3 ${ }^{+}$, Fgfr4 $4^{-}$cells are present, notably dorsally, consistent with the absence of Fgfr4 expression in the Pax3-positive cells of the dermomyotome, which is beginning to disaggregate at E10.5 (Relaix et al. 2005). When we looked at $\beta$-galactosidase versus Fgfr4 expression on sections of transgenic embryos expressing the Fgfr $4(559 \mathrm{bp})$ tk-nlacZ transgene, we detected colocalization in cells in the myotome at E11.5 (Fig. 3I,J), demonstrating that the 559-bp sequence reflects Fgfr4 expression. Comparison of Pax3 and $\beta$-Gal labeling of transgenic embryos in muscle masses of the trunk shows colocalization at E11.5 (Fig. 3K) in 18\% of $\beta$-Gal-positive cells, estimated across several sections, whereas at E10.5, this percentage is higher (43\%) (Fig. 3L). A similar developmental difference was seen for the forelimbs (Fig. 3M,N). This correlates with the arrival of Pax3/7-positive cells in the somite or limbs, which subsequently express myogenic factors and differentiate, and might suggest that this transgene is also dependent on factors other than Pax3.

The 559-bp element also contains E-box consensus sites (Fig. 2D, E1-E4) for the myogenic factor family of transcription factors. To investigate their potential involvement, we examined genetically the relationship be- 
A

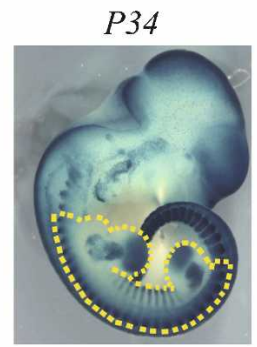

E11.5
B

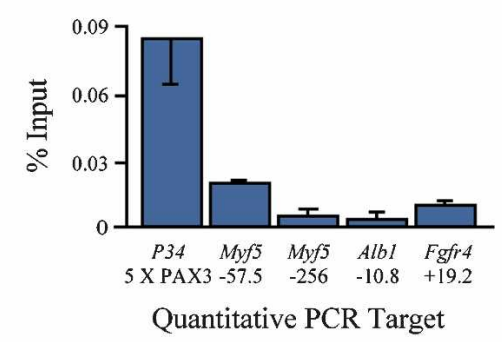

C

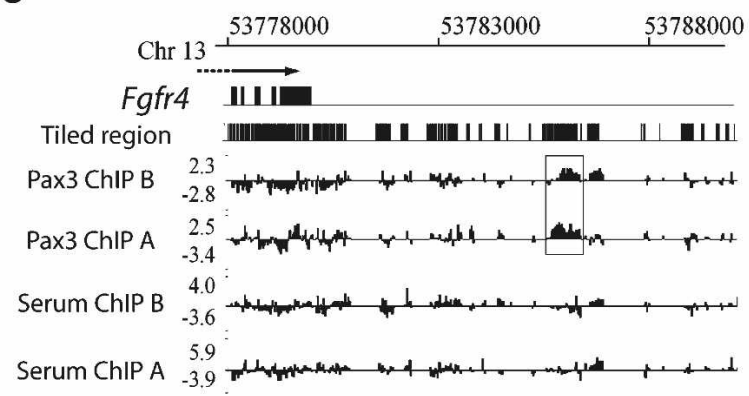

D
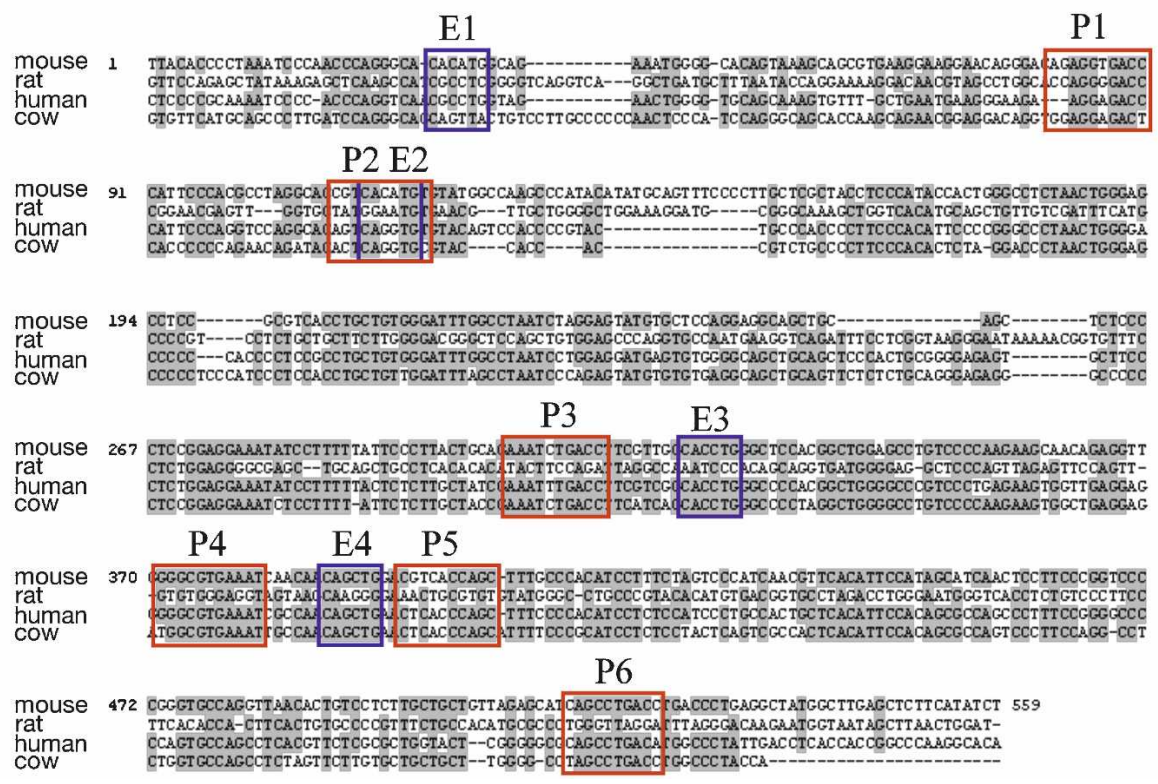

E

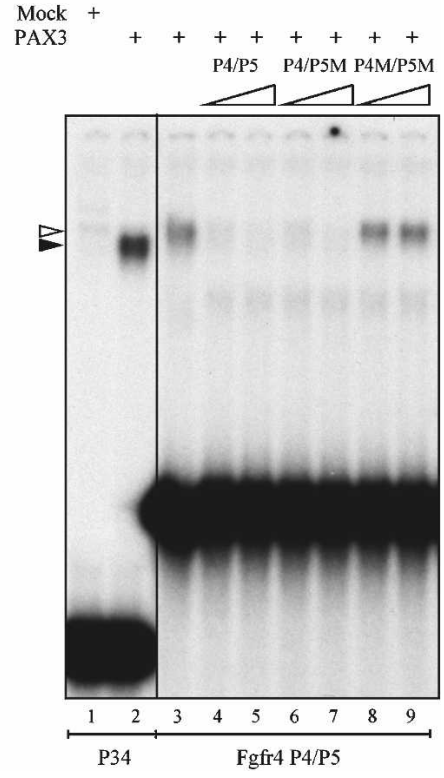

Figure 2. Identification of an in vivo Pax3-binding site in the Fgfr4 locus. (A) X-Gal staining at E11.5 of a $P 34$ transgenic embryo in which nlacZ expression is regulated by five consensus Pax3-binding sites $(\text { Pax } 3)_{5}-t k$-nlacZ. The tissue used for ChIP is outlined. (B) Real-time quantitative PCR using primers (targets) for the P34 Pax3-binding sites, a functional Pax3 site at $-57.5 \mathrm{~kb}$ from Myf5, a control Myf5 flanking sequence, a control Albumin sequence, and the Fgfr4 +19.2-kb sequence, containing sites P4/P5 identified by Chip-chip. Results are expressed as a percentage of input showing enrichment after Pax3 immunoprecipitation, with the serum control subtracted. $(C)$ Results of the tiling arrays for Pax3 and for serum controls within the 3' part of the Fgfr 4 locus. Fgfr 4 exons are indicated as black boxes. The region of strong Pax3 hybridization signal is outlined. (D) The nucleotide sequence of the $F g f r 4(+18,832$ to $+19,391$ bp) distal element in mouse and comparison with a homologous region of the rat, human, and cow genomes, with conserved bases indicated on a gray background. Six putative Pax3 binding sites (P1-P6) are framed in red. E-box consensus sequences for myogenic regulatory factors are also indicated in blue (E1-E4). (E) Gel shift mobility assays for Pax3 binding, using a reticulocyte lysate without (lane 1) or with (lane 2-9) Pax3 protein. A labeled oligonucleotide (30 bp) containing a consensus Pax3 site of the P34 transgene (lanes 1,2) or an oligonucleotide (60 bp) from the Fgfr4 (559 bp) sequence containing sites P4 and P5 (lanes 3-9) shows Pax3 binding (3,8,9). Lanes 4-9 show competition experiments with 50-fold (lane 4) or 150-fold (lane 5) excess of wild-type sequence (P4/P5), with this sequence with P5 mutated (P4/P5M) (lanes 6,7) or with P4 and P5 mutated (P4M/P5M) (lanes 8,9).

tween MyoD, Myf5, and Fgfr4. Expression of Fgfr4 in Myf5 $5^{\text {nlacZ/nlacz }}$ mutant embryos is absent, until MyoD is activated later and the myotome begins to form (Supplemental Fig. S2), consistent with Fgfr4 expression in cells of the myotome. This might also suggest direct regulation by MyoD, although in the MyoD mutant, Fgfr4 is normally expressed at E10.5 and E11.5 (data not shown); however, in the absence of MyoD, Myf5 may act on Fgfr4. The primary importance of Pax3 in Fgfr4 expression is supported by the $\mathrm{Pax} 3^{\mathrm{Pax} 3-\mathrm{En} /+}$ mouse. When Pax3 is compromised, Fgfr4 expression is down-regulated (Fig.
1; Supplemental Fig. S3D), whereas $M y f 5 / M y o D$ continue to be expressed in most of the myotome (Supplemental Fig. S3E,F).

We mutated the four E-boxes in the 559-bp element. Since E2 is close to P2 (Fig. 2D), we took care not to interfere with this Pax-binding site, and similarly when P2 was mutated, the E2 site was kept intact. These E-box mutations result in reduced expression of the transgene in the trunk, whereas expression in more anterior muscles and in the limbs is not so notably affected (Fig. $4 C, D)$. Expression is not detected in myotomes of imma- 

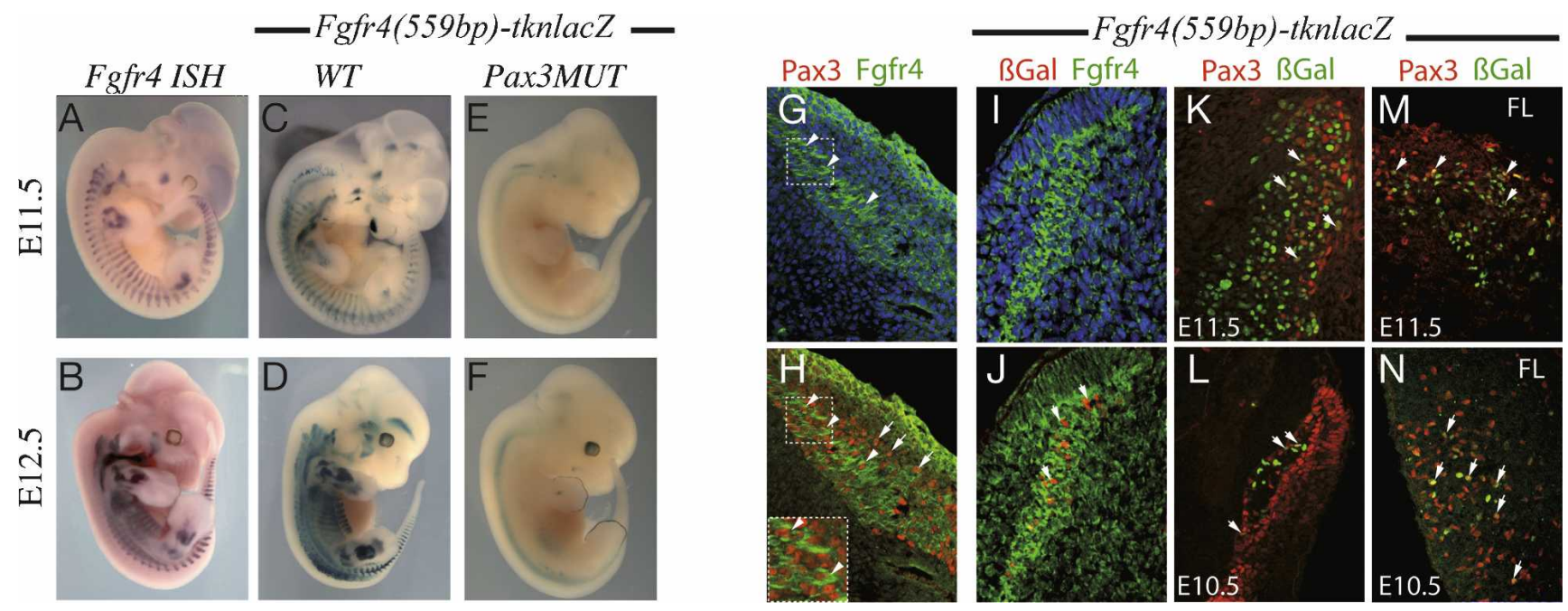

Figure 3. The Fgfr4 distal element directs Pax3-dependent myogenic expression in vivo. $(A, B)$ Whole-mount in situ hybridization for Fgfr4 transcripts on embryos at the stages indicated. $(C-F)$ X-Gal staining of transient transgenic embryos in which a tk-nlacZ transgene is under the control of the wild-type $(C, D)$ Fgfr4 distal element ( $559 \mathrm{bp}$ ) or this element with the six Pax3 sites, mutated $(E, F)$. $(G, H)$ Coimmunohistochemistry on DAPI-stained transverse sections in the myotome at interlimb level of E10.5 embryos, showing Fgfr4 (green) and DAPI staining $(G)$ and Fgfr4 (green) and Pax3 (red) staining $(H)$. Insert in $H$ represents a higher magnification of the region outlined in $G$ and $H$. Examples of colocalization where the plane of section includes a nucleus showing Pax3 staining are indicated by arrowheads. Arrows indicate dorsally located $\mathrm{Pax}^{+}$cells. $(I, J)$ Coimmunohistochemistry on DAPI-stained sections $(I)$ at trunk level of an E11.5 Fgfr4(559bp)-tk-nlacZ transgenic embryo using antibodies to Fgfr4 (green) (I,J) and to nuclear $\beta$-Gal (red) (J). Arrowheads as for $H .(K, L)$ Coimmunohistochemistry on transverse sections at interlimb level on an E11.5 $(K)$ or E10.5 $(L)$ Fgfr4(559bp)-tk-nlacZ transgenic embryo showing expression of Pax3 (red) and $\beta$-Gal (green). (M,N) Sections of the forelimb (FL) at E11.5 $(M)$ or E10.5 $(N)$ similarly stained for Pax3 and $\beta$-Gal. Arrows point to nuclear coexpression.

ture posterior somites, whereas variable partial expression is seen in more mature muscle masses in the trunk. This suggests that Fgfr4 expression in the myotome, which is Pax3-dependent (Fig. 3C-F), is also E-box-dependent. Immunostaining on sections, at E11.5, shows partial colocalization of MyoD with reporter $\beta$-Gal from the transgene in myogenic cells in both trunk and forelimbs $(\sim 50 \%)$ (Fig. 4E,F).

\section{Pax3 regulation of the FGF signaling pathway and its impact on myogenesis}

Other components of the FGF signaling pathway are also potential Pax3 targets. One such sequence is Sprouty1, which is up-regulated in $P a \times 3^{P A X 3-F K H R / G F P}$ embryos (data not shown) and down-regulated in the somites of $\mathrm{Pax}^{\mathrm{Pax} 3-\mathrm{En} /+}$ embryos (Fig. 5A,B). As in Figure 1, X-Gal staining of the somites confirms that the cells that normally express Pax3 are present (Fig. 5A', $\mathrm{B}^{\prime}$ ). Sprouty 1 transcripts are detected in Pax3-positive progenitor cells at the edges of the dermomyotome, as well as in the myotome (Fig. 5A; Supplemental Fig. S4).

Sprouty1, which modulates the FGF signaling pathway (Hacohen et al. 1998), is reduced in Pax3-GFP-positive cells isolated by flow cytometry from interlimb somites of $P a \times 3^{G F P / n l a c Z}$-null embryos (Fig. 5C). This is also the case for the FGF signaling intermediates, phospho-AKT and phospho-p38, whereas phospho-ERK is upregulated (Fig. 5C), showing the in vivo impact of Pax3 on the FGF pathway. Up-regulation of phospho-ERK in the $P a \times 3^{-/-}$somitic cells probably reflects the downregulation of Sproutyl that is known to negatively regulate phospho-ERK (Hanafusa et al. 2002).

In order to look at FGF function during myogenesis in the mouse embryo, we perturbed FGF signaling by manipulating Sprouty expression. This strategy avoids potential problems due to the presence of other FGF receptors that may compensate for loss of Fgfr4 function in the Fgfr4 mutant. A transgenic line that expresses Sprouty2 under the control of a CAG promoter (Sprouty2GOF) (Calmont et al. 2006; Basson et al. 2008) was targeted to Pax3-expressing cells and their derivatives by crossing with a $\mathrm{Pax} 3^{\mathrm{Cre} /+}$ line (Engleka et al. 2005). In this experiment, because of transgene availability, Sprouty2 was manipulated; however, Sprouty1 and Sprouty2 are thought to have equivalent functions (Kim and Bar-Sagi 2004; Taketomi et al. 2005). Expression of the transgene reduces phospho-ERK levels, as normalized to $\beta$-tubulin (Fig. 5D). Sprouty2 was clearly expressed at sites of myogenesis, as indicated by alkaline phosphatase staining from the transgene reporter (Calmont et al. 2006; data not shown). We analyzed the number of Pax7- versus myogenin (Myog)-positive cells on serial sections of trunk and limb muscles at E13.5 (Fig. 5E,F). At this stage, Pax7 is coexpressed with Pax3 in trunk and limb muscle progenitor cells. This ratio varies across each muscle mass, but we observed a consistent bias toward Pax7 in the Sprouty gain of function embryos as shown quantitatively for back muscles in Figure 5G. 


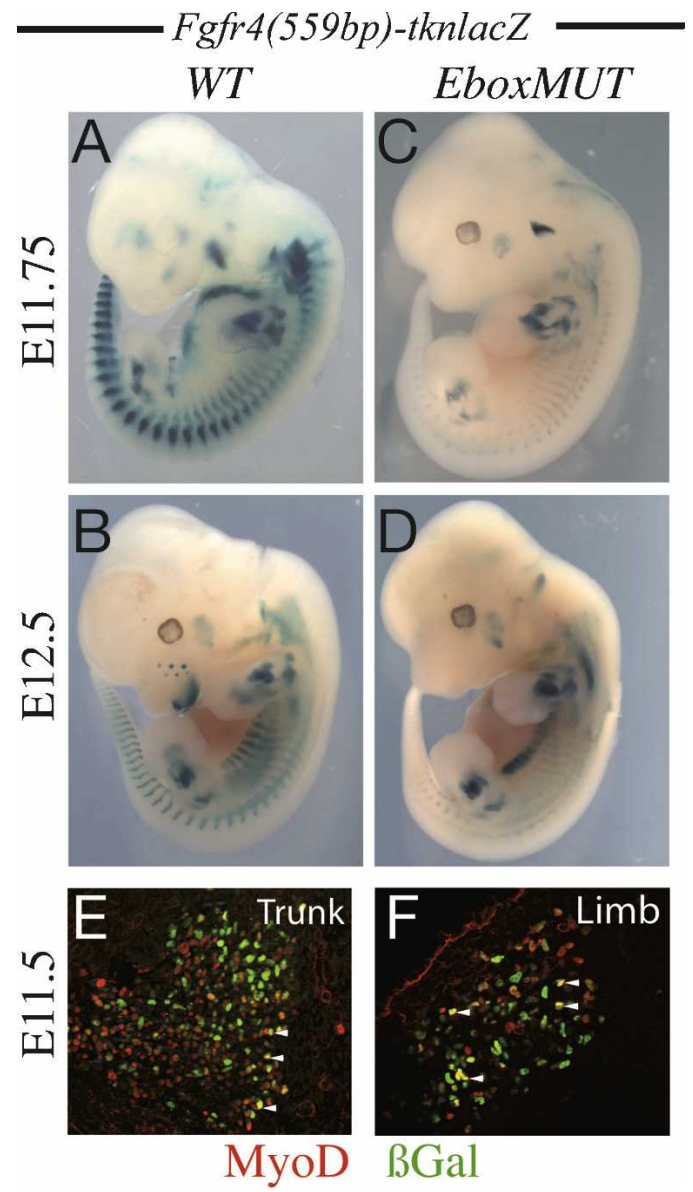

Figure 4. Potential myogenic factor regulation of the Fgfr4 element. $(A, B)$ Control embryos expressing the Fgfr4(559bp)-tknlacZ transgene at E11.75 $(A)$ and E12.5 $(B) .(C, D)$ Embryos expressing this transgene with the four E-boxes mutated at E11.75 $(C)$ and E12.5 $(D) .(E, F)$ Coimmunohistochemistry on DAPIstained transverse sections of Fgfr4(559bp)-tk-nlacZ transgenic embryos at E11.5 in the interlimb region (Trunk) (E) and fore$\operatorname{limb}(\operatorname{Limb})(F)$ using antibodies to $\mathrm{MyoD}$ (red) and $\beta$-Gal (green). Arrowheads point to examples of colocalization.

\section{Discussion}

Genetic data indicated that Fgfr4 lies genetically downstream from Pax3, and following on this, we show that the Fgfr4 gene is a direct target of Pax3. Our ChIP-onchip experiments led us to the identification of a novel myogenic regulatory element in the 3 ' flanking region of the gene. The Pax3-binding sites identified in the 559-bp sequence are also putative Pax7-binding sites, since Pax3 and Pax7 exhibit similar DNA-binding characteristics and activities (Relaix et al. 2004). Thus Fgfr4 expression at sites of myogenesis in the head, where Pax3 is absent, may be regulated by $\operatorname{Pax} 7$.

The Fgfr4 transgene is expressed in a proportion of Pax3/7-positive cells that decreases as the muscle masses develop. Its activation in these cells may also depend on other upstream regulators of myogenesis, and, indeed, the 559-bp element also contains a Six-binding site. Activation of the $-57.5-\mathrm{kb} M y f 5$ enhancer depends on $\mathrm{Pax} 3 / 7$, but it is also regulated by Six $1 / 4$ (Giordani et al. 2007), and this may be the case for Fgfr4.

The 559-bp element directs transgene expression in MyoD-positive myogenic cells as well as in progenitors, and mutation of E-boxes present in the sequence reduces its expression. Functional analysis in transgenic embryos demonstrates that the Pax3 sites are required for transcription. Pax3 therefore acts directly on the 559-bp element, rather than exerting its effects indirectly via the myogenic regulatory factors. In keeping with this, when Pax3 activity is compromised in $\mathrm{Pax}^{\text {Pax } 3-E n /+}$ embryos, the endogenous Fgfr4 gene is down-regulated despite the continuing expression of Myf5 and MyoD in most of the myotome. It is notably in the limbs, where Myf5 is directly regulated by Pax3 (Bajard et al. 2006), that mutation of the E-boxes has the least effect. In Fgfr4(559bp)-tknlacZ transgenic embryos, the progressive maturation of somites and their muscle derivatives along the anterior/posterior axis permits visualization of the role of the E-boxes at different developmental stages within the same embryo (see Fig. 4). Expression of the transgene at the onset of myogenesis in the most posterior somites is severely affected in the absence of E-boxes, indicating that this requires myogenic factors as well as Pax3, present in the cells that delaminate from the edges of dermomyotome to form the early myotome (Buckingham and Relaix 2007). In more mature anterior somites, where a Pax3/7-positive population of progenitor cells has invaded the myotome as a result of de-epithelialization of the central dermomyotome, the E-box requirement is rather less striking. In interlimb somites at E10.5, when this invasion is initiated, many Pax3/7positive cells express the transgene, whereas this number is lower at E11.5. During this later phase of myogenesis (Buckingham and Relaix 2007), transgene expression may therefore be initiated in Pax3/7-positive cells and only subsequently come under myogenic factor regulation, which provides a means of maintaining Fgfr4 expression in differentiating muscle cells in which Pax $3 / 7$ are down-regulated. Although the transgene is expressed in MyoD-positive cells in the limbs, E-box dependence at this site is much less evident, providing another example of differences in the regulatory circuits operating in limb versus trunk myogenesis (Relaix et al. 2004).

Interference with FGF signaling, through artificially increased levels of Sprouty, tends to prevent Pax3/7-positive myogenic progenitor cells from entering the skeletal muscle differentiation program. This is consistent with observations in the zebrafish embryo, where interference with Fgf8 (a potential ligand of Fgfr4) (MacArthur et al. 1995) leads to an increase in Pax3/7-positive progenitor cells in somites (Hammond et al. 2007). Results obtained in chick head muscles also suggest that the FGF pathway, mediated via Fgf8, can influence the ratio between proliferative progenitors (in this case, $\mathrm{MyoR}^{+}$) and differentiated cells $\left(\mathrm{Myf}^{+}\right)$(von Scheven et al. 2006). Pax3 activation of Fgfr4 will promote myogenesis, and, indeed, down-regulation of this receptor was found to adversely affect muscle differentiation in the chick embryo (Edom-Vovard et al. 2001; Marics et al. 2002). However, 

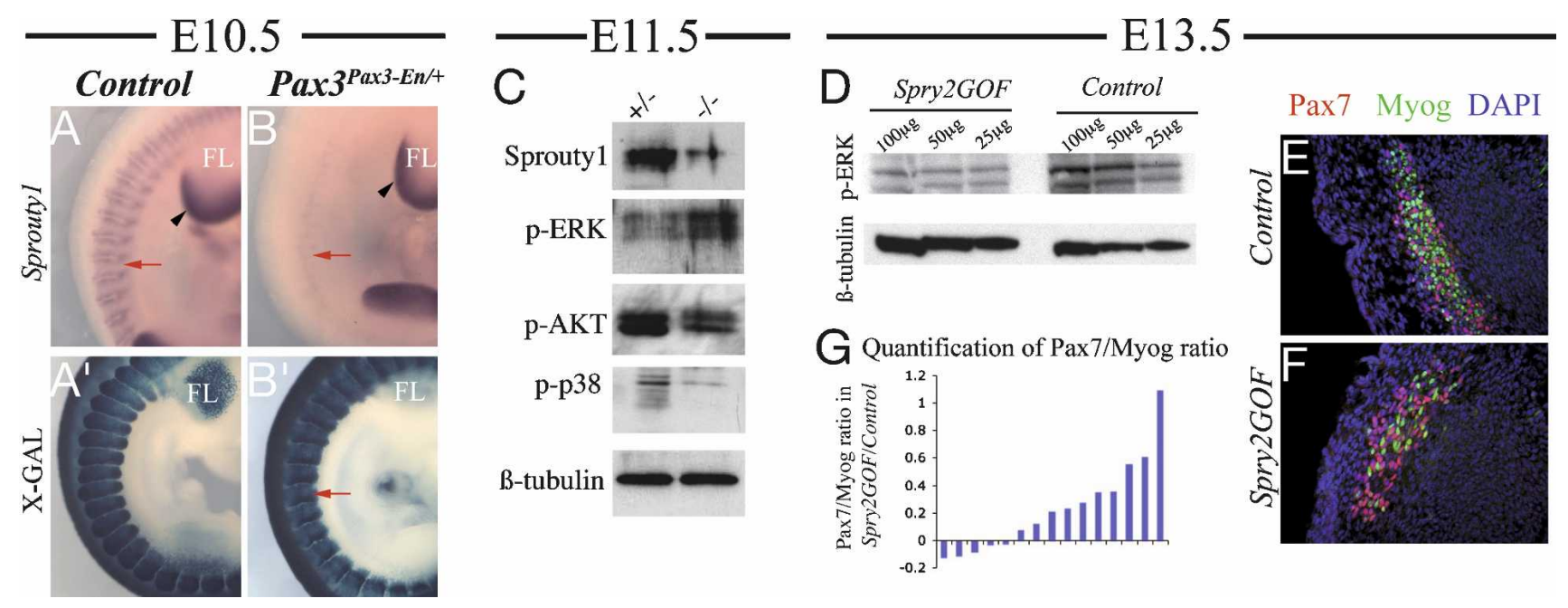

Figure 5. Components of the FGF signaling pathway are regulated by Pax3; Sprouty modulates myogenesis in vivo. $(A, B)$ Wholemount in situ hybridization for Sprouty 1 transcripts in control $(A)$ andPax3 $3^{\text {Pax3-En/+ }}(B)$ embryos at E10.5. $\left(A^{\prime}, B^{\prime}\right) \mathrm{X}^{-G a l}$ staining of $\operatorname{Pax}^{\text {nlacZ/+ }}\left(A^{\prime}\right)$ and $\operatorname{Pax}^{\text {Pax3-En/+ }}\left(B^{\prime}\right)$ embryos at E10.5. The red arrows indicate somites. Black arrowheads point to Pax3-independent Sprouty1 expression in the distal forelimb bud (FL). (C) Western blot of the same number of GFP-positive cells isolated by flow cytometry, from somites of $P a x 3^{G F P /+}(+/-)$ and Pax3 ${ }^{G F P / n l a c Z}(-/-)$ embryos at E1 1.5, using the antibodies indicated; (p) phosphorylated. (D) Western blots on extracts $(100 \mu \mathrm{g}, 50 \mu \mathrm{g}$, and $25 \mu \mathrm{g}$ of total protein) from limbs of control and Sprouty2 gain of function (Spry2 GOF) transgenic embryos at E13.5, using the antibodies indicated. $(E, F)$ Coimmunohistochemistry on DAPI-stained transverse sections of equivalent muscles in the interlimb region of control $(E)$ and Spry2 GOF $(F)$ embryos at E13.5 using antibodies to Pax7 (red) and myogenin (MyoG) (green). (G) Quantitative analysis of the ratio of Pax7 to myogenin-positive cells on sections, counted using Metamorph software. Each bar represents the differential ratio $\left[(\operatorname{Pax} 7 / \mathrm{Myog})_{\text {Spry2GOF }}-(\operatorname{Pax} 7 / \mathrm{Myog})_{\text {control }}\right]$ between Spry2GOF and control embryos quantified for equivalent sections from the same deep back muscles, with a minimum of 500 total cells counted per section. The ratio (Pax7/Myog) is significantly higher in Spry2GOF samples compared to the control $(P<0.05$, with the two-sided Wilcoxon signed rank test).

we show that Sprouty1 also lies genetically downstream from $P a x 3$, and preliminary results suggest that it may also be a direct Pax3 target. In the mouse embryo, the transcription of Sprouty1 in Pax3-positive progenitor cells at the edges of the dermomyotome, as well as in the myotome, suggests a model in which Sproutyl dampens premature myogenesis. Such an interplay between Sproutyl and Fgfr4 in myogenic progenitors is consistent with a recent transcriptome analysis on quiescent and activated satellite cells in adult muscle (Fukada et al. 2007), which shows that Sprouty1 transcripts are restricted to the former, whereas Fgfr 4 transcripts are also present in the activated cells, which now express $M y o D$ (Kastner et al. 2000). Fgfr4 expression has also been reported in regenerating muscle after injury (Zhao and Hoffman 2004), and in Fgfr4-null mice, muscle regeneration is affected (Zhao et al. 2006). In this model, it has been suggested that MyoD affects Fgfr4 expression through Tead2 acting on the promoter. It remains to be seen whether the 559-bp element is also active in Pax7positive satellite cells of adult muscle.

During myogenesis in the embryo, we propose that Pax3, acting directly on Fgfr4 and on Sprouty1, orchestrates the potential effects of FGF signaling on the formation of skeletal muscle. We showed previously that Pax3 directly activates the myogenic determination gene Myf5 (Bajard et al. 2006). Pax3 regulation of FGF signaling now provides a further level of myogenic control by governing the balance between stem cell maintenance and tissue differentiation.

\section{Material and methods}

Preparation of embryos

Embryos were collected after natural overnight matings, and dated taking E0.5 as the day after the vaginal plug.

$X$-Gal staining, immunohistochemistry, and whole-mount in situ hybridization

$\mathrm{X}$-Gal staining was performed as described in Bajard et al. (2006).

Whole-mount in situ hybridization with digoxigenin-labeled probes was performed as described in Tajbakhsh and Cossu (1997). The mouse Fgfr4 probe was a gift from Christophe Marcelle.

The Sprouty1 probe was cloned by PCR using the following primers: SprylA (5'-AGAATTCCACATGGATTCCCCAAGT CAGCATGGC-3') and Spry1B (5'-AAAGGCC AAGAGCCAA AGGCACCTTAGGGAGCTG-3').

Coimmunofluorescence on sections was carried out as previously described (Relaix et al. 2003) except for Pax7 and Fgfr4 antibodies, where an antigen retrieval procedure was used (Gayraud-Morel et al. 2007). The following antibodies were used: anti-Pax3 (monoclonal, DSHB, 1/250), anti-Pax7 (monoclonal, DSHB, 1/200), anti-MyoD (monoclonal, DAKO, 1/200), antimyogenin (polyclonal, Santa Cruz, $1 / 200$ ), anti- $\beta$-galactosidase (polyclonal, provided by J.-F. Nicolas, 1/300), and anti-Fgfr4 
(polyclonal, Santa Cruz Biotechnologies, 1/250). Secondary antibodies were coupled to fluorochromes: Alexa 488 (Molecular Probes, 1/500) and Alexa 546 (Molecular Probes, 1/1500). Images were obtained with an Apotome Zeiss microscope and Axiovision software. All images were assembled in Adobe Photoshop.

\section{Plasmid constructions used for transgenesis}

To generate the Fgfr4(559bp)-tk-nlacZ transgene, the 559-bp Fgfr4 element (EU697430) was synthesized by PCR using an Expand High Fidelity kit (Roche). The forward and reverse primers used were: fwd (5'-TTACACCCCTAAATCCCAACCCAG$\left.3^{\prime}\right)$ and rev (5'-AAGGGTGACTTGGAGGTTCCAGAG-3'). After sequence verification, the PCR product was cloned into a pGEM-T easy vector (Promega), and the 559-bp NotI-NotI fragment was then cloned into the NotI site of the $t k-n l a c Z$ plasmid (Hadchouel et al. 2000). Mutagenesis was performed using the QuickChange Multi Site-Directed Mutagenesis kit (Stratagene) using as a matrix Fgfr4(559bp) subcloned into pGEM-T easy plasmid (Promega). The TGAC core sequence of the Pax 3 consensus binding sites was replaced by gtct (Bajard et al. 2006) except for sites 2 and 5, in which the consensus was mutated into atgt to avoid recreating a potential Pax site. The CA(nn)TG core sequence of the E-box consensus was replaced by $\mathrm{Cg}(\mathrm{nn}) \mathrm{aG}$ except for E-box2, in which the consensus has been replaced by $\mathrm{CA}(\mathrm{nn}) \mathrm{Ta}$ to avoid affecting the Pax3 site, P2. Mutation of the Pax3 site, P2, has no effect on the E2 sequence.

\section{Generation of transgenic embryos}

Plasmid fragment purification and transgenic embryos were generated as described previously (Hadchouel et al. 2000). Transient transgenic embryos were dated taking the day of reimplantation as E0.5. With the unmutated Fgfr4(559bp)-tk-nlacZ sequence, 5/5 transgenic embryos at E11.5 and 5/5 at E12.5 expressed the transgene at sites of myogenesis. With the Pax 3 sites mutated, myogenic expression was very strongly reduced or absent (0/4 at E11.5 and 0/5 at E12.5). With the four E-box sites mutated, myogenic expression in limb buds was observed in $3 / 3$ at E11.5, and 3/3 at E12.5, transient transgenic embryos, while expression in the trunk was reduced in all transgenic embryos.

\section{Western blots on embryonic tissues}

Somites from four $\mathrm{Pax} 3^{\mathrm{GFP} /+}$ and five $\mathrm{Pax} 3^{\mathrm{GFP} / \text { nlacZ }}$ mutant embryos at E11.5 were dissected in DMEM medium under a fluorescent microscope for each experiment. Material was dissociated with a $1-\mathrm{mL}$ syringe and then filtered. After flow cytometry sorting, $5 \times 10^{5}$ cells were collected from both genotypes, and extracts were prepared and analyzed by Western blotting as described previously (Lindon et al. 2000). The following antibodies were used: anti-Sprouty1, Zymed, 1/1000; anti-phospho-p44/42 Map Kinase (p-ERK), Cell Signaling, 1/1000; anti-phospho-AKT (p-AKT), Cell Signaling, 1/750; anti-phospho-p38 (p-p38), Sigma, $1 / 750$; and anti- $\beta$-tubulin, Chemicon, $1 / 2000$.

\section{ChIP}

Somites and limb buds were dissected from 110 P34 transgenic embryos at E11.5. ChIP was performed as described previously (Wiebe et al. 2007) with the following modifications. Chromatin was sheared to a range of 0.1 to $1 \mathrm{~kb}$. The equivalent of $150 \mu \mathrm{g}$ of chromatin was used in each immunoprecipitation experiment and immunoprecipitated with $2 \mu \mathrm{L}$ of Pax3 whole-serum antibody (Geneka) or $2 \mu \mathrm{L}$ of Normal Rabbit Serum (Chemicon).
Purified immunoprecipitated DNA was resuspended into 200 $\mu \mathrm{L}$ of distilled water. The equivalent of $10 \%$ of input chromatin was DNA purified in parallel and resuspended in an equal volume.

\section{Real-time PCR}

Four microliters of ChIP product were used in quantitative PCR experiments. A serial 1/10 dilution series of the $10 \%$ input chromatin was used to develop a standard curve for calculating the percent input for each ChIP sample.

The following primers were used: Pax3-binding sites of the P34 transgene, P34fwd (5'-AGGAATTCGATCCCTCGT CACG-3'), P34rev (5'-AGCTTGATGGACATTCGAAGCG-3'); the -57.5 -kb Myf5 element, -57.5fwd (5'-TGTCCCCATATTA CTTCCCAAGCT-3'), -57.5rev (5'-TCTCCGTATGTTTGTTG GAAGG-3'); Albumin enhancer, Albfwd (5'-GGGACGAGA TGGTACTTTGTG-3'), Albrev (5'-GATCAGTCCAAACTTCT TTCTG-3'). For the Myf5 200-kb flanking sequence, primers previously described were used (Bajard et al. 2006). The Fgfr4 50 mer showing the strongest hybridization signal in ChIPchip is located at $+19.2 \mathrm{~kb}$ and encompasses sites P4/P5, Fgfr4 $+19.2 \mathrm{kbrev} \quad\left(5^{\prime}\right.$-CACCAGCTTTGCCCACATC-3'), Fgfr4 +19.2kbfwd (5'-GGGACCGGGAAGGAGTTG-3').

\section{Ligation-mediated PCR and ChIP-on-chip}

Fifty microliters of the ChIP product DNA (i.e., $25 \%$ of the original material) were first purified using a QIAquick PCR purification column following the manufacturer's instructions (Qiagen). Ligation-mediated PCR of ChIP and input material was performed as in the NimbleGen protocol. The amplified material was analyzed using a 2100 Bioanalyzer with a dsDNA 12,000 laboratory chip (Agilent Technologies), and displayed an average size of $300 \mathrm{bp}$ with a range of 150 to $900 \mathrm{bp}$. Four micrograms of each sample were sent to Nimblegen for labeling and hybridization to custom microarrays. The microarrays had 210 loci, tiled at a density of one 50-nucleotide probe per $24 \mathrm{bp}$. Each locus on the array was repeat-masked and contained the entire coding region as well as $30 \mathrm{~kb}$ upstream and $10 \mathrm{~kb}$ downstream. Further details of the design of the NimbleGen custom array will be published elsewhere. Pax3 antibody ChIP were hybridized with input chromatin, and control antibody ChIP products were hybridized with input chromatin from each of the biological replicates A and B.

\section{Fgfr4 distal element identification and Pax3 site selection}

The Fgfr4 transcript and coding sequence was based on the Ensembl ENMUST00000005452 transcript and ENSMUG00000005320 gene sequence. The University of California at Santa Cruz Genome Browser was used to predict a 559-bp region surrounding the Pax3-binding sites that was highly conserved in mammals, and sequences from the mouse, human, rat, and cow were aligned with ClustalW. Bases that were conserved between the species were shaded, with a minimum of three out of four base matches required for shading (Fig. 2D).

\section{Electrophoretic mobility shift assay (EMSA)}

Pax3 synthesis with a reticulocyte lysate and EMSA were carried out as described previously in Bajard et al. (2006).

Probe sequences were as follows, with Pax3 sites underlined: the Pax3-binding site of $P 34\left(5^{\prime}\right.$-TCGATCCCTCGTCACG CTTCGAATGTCCAT-3'), Fgfr4P4/P5 (5'-AACAGAGGTTG GGGCGTGAAATCAACAACAGCTGGACGTCACCAGCTT 
TGCCCACATC-3'), Fgfr4P4/P5M (5'-AACAGAGGTTGGGG CGTGAAATCAACAACAGCTGGACtatgCCAGCTTTGCCC ACATC-3'), Fgfr4P4M/P5M, where both Pax3 sites have been mutated (5'-AACAGAGGTTGGGGCGgtccATCAACAACAG CTGGACtatgCCAGCTTTGCCCACATC-3').

\section{Acknowledgments}

We thank C. Bodin, A. Cumano, Y. Watanabe, and P. Daubas for their contributions and the Dynamic Imaging platform (IP) for help with image acquisition; and especially A. Danckaert for image quantification with Metamorph software. We are grateful to C. Marcelle for stimulating discussions and the gift of the Fgfr4 probe and G. Martin for sharing the Sprouty2GOF transgenic line. The work in M.B.'s laboratory was supported by the Pasteur Institute and the CNRS and by grants from the AFM and the E.U., through the Integrated Projects EuroStemCell, EuroSyStem, and the Networks of Excellence, Cells into Organs, and MYORES. We also acknowledge support from the Canceropole IDF (to F.R), NIH R37GM36477 (to K.S.Z.), the Mathers Charitable Foundation (to K.S.Z.), and the NCI CA06927 (to K.S.Z.). M.L. was supported by fellowships from the French Ministry of Education and Research and the AFM.

\section{References}

Bajard, L., Relaix, F., Lagha, M., Rocancourt, D., Daubas, P., and Buckingham, M.E. 2006. A novel genetic hierarchy functions during hypaxial myogenesis: Pax3 directly activates Myf5 in muscle progenitor cells in the limb. Genes \& Dev. 20: 2450-2464.

Basson, M.A., Echevarria, D., Petersen Ahn, C., Sudarov, A., Joyner, A.L., Mason, I.J., Martinez, S., and Martin, G.R. 2008. Specific regions within the embryonic midbrain and cerebellum require different levels of FGF signaling during development. Development 135: 889-898.

Ben-Yair, R. and Kalcheim, C. 2005. Lineage analysis of the avian dermomyotome sheet reveals the existence of single cells with both dermal and muscle progenitor fates. Development 132: 689-701.

Bladt, F., Riethmacher, D., Isenmann, S., Aguzzi, A., and Birchmeier, C. 1995. Essential role for the c-met receptor in the migration of myogenic precursor cells into the limb bud. Nature 376: 768-771.

Buckingham, M. 2006. Myogenic progenitor cells and skeletal myogenesis in vertebrates. Curr. Opin. Genet. Dev. 16: 525532.

Buckingham, M. and Relaix, F. 2007. The role of Pax genes in the development of tissues and organs: Pax3 and Pax7 regulate muscle progenitor cell functions. Annu. Rev. Cell Dev. Biol. 23: 645-673.

Calmont, A., Wandzioch, E., Tremblay, K.D., Minowada, G., Kaestner, K.H., Martin, G.R., and Zaret, K.S. 2006. An FGF response pathway that mediates hepatic gene induction in embryonic endoderm cells. Dev. Cell 11: 339-348.

Edom-Vovard, F., Bonnin, M.A., and Duprez, D. 2001. Misexpression of Fgf-4 in the chick limb inhibits myogenesis by down-regulating Frek expression. Dev. Biol. 233: 56-71.

Engleka, K.A., Gitler, A.D., Zhang, M., Zhou, D.D., High, F.A., and Epstein, J.A. 2005. Insertion of Cre into the Pax3 locus creates a new allele of Splotch and identifies unexpected Pax3 derivatives. Dev. Biol. 280: 396-406.

Epstein, J.A., Shapiro, D.N., Cheng, J., Lam, P.Y., and Maas, R.L. 1996. Pax3 modulates expression of the c-Met receptor dur- ing limb muscle development. Proc. Nat1. Acad. Sci. 93: 4213-4218.

Fukada, S., Uezumi, A., Ikemoto, M., Masuda, S., Segawa, M., Tanimura, N., Yamamoto, H., Miyagoe-Suzuki, Y., and Takeda, S. 2007. Molecular signature of quiescent satellite cells in adult skeletal muscle. Stem Cells 25: 2448-2459.

Gayraud-Morel, B., Chretien, F., Flamant, P., Gomes, D., Zammit, P.S., and Tajbakhsh, S. 2007. A role for the myogenic determination gene Myf5 in adult regenerative myogenesis. Dev. Biol. 312: 13-28.

Giordani, J., Bajard, L., Demignon, J., Daubas, P., Buckingham, M., and Maire, P. 2007. Six proteins regulate the activation of Myf5 expression in embryonic mouse limbs. Proc. Natl. Acad. Sci. 104: 11310-11315.

Hacohen, N., Kramer, S., Sutherland, D., Hiromi, Y., and Krasnow, M.A. 1998. sprouty encodes a novel antagonist of FGF signaling that patterns apical branching of the Drosophila airways. Cell 92: 253-263.

Hadchouel, J., Tajbakhsh, S., Primig, M., Chang, T.H., Daubas, P., Rocancourt, D., and Buckingham, M. 2000. Modular long-range regulation of Myf5 reveals unexpected heterogeneity between skeletal muscles in the mouse embryo. Development 127: 4455-4467.

Hammond, C.L., Hinits, Y., Osborn, D.P., Minchin, J.E., Tettamanti, G., and Hughes, S.M. 2007. Signals and myogenic regulatory factors restrict pax3 and pax7 expression to dermomyotome-like tissue in zebrafish. Dev. Biol. 302: 504521.

Hanafusa, H., Torii, S., Yasunaga, T., and Nishida, E. 2002. Sprouty1 and Sprouty2 provide a control mechanism for the Ras/MAPK signalling pathway. Nat. Cell Biol. 4: 850-858.

Kastner, S., Elias, M.C., Rivera, A.J., and Yablonka-Reuveni, Z. 2000. Gene expression patterns of the fibroblast growth factors and their receptors during myogenesis of rat satellite cells. J. Histochem. Cytochem. 48: 1079-1096.

Kim, H.J. and Bar-Sagi, D. 2004. Modulation of signalling by Sprouty: A developing story. Nat. Rev. Mol. Cell Biol. 5: 441-450.

Klein, O.D., Lyons, D.B., Balooch, G., Marshall, G.W., Basson, M.A., Peterka, M., Boran, T., Peterkova, R., and Martin, G.R. 2008. An FGF signaling loop sustains the generation of differentiated progeny from stem cells in mouse incisors. Development 135: 377-385.

Lindon, C., Albagli, O., Domeyne, P., Montarras, D., and Pinset, C. 2000. Constitutive instability of muscle regulatory factor Myf5 is distinct from its mitosis-specific disappearance, which requires a D-box-like motif overlapping the basic domain. Mol. Cell. Biol. 20: 8923-8932.

MacArthur, C.A., Lawshe, A., Xu, J., Santos-Ocampo, S., Heikinheimo, M., Chellaiah, A.T., and Ornitz, D.M. 1995. FGF-8 isoforms activate receptor splice forms that are expressed in mesenchymal regions of mouse development. Development 121: 3603-3613.

Marcelle, C., Wolf, J., and Bronner-Fraser, M. 1995. The in vivo expression of the FGF receptor FREK mRNA in avian myoblasts suggests a role in muscle growth and differentiation. Dev. Biol. 172: 100-114.

Marics, I., Padilla, F., Guillemot, J.F., Scaal, M., and Marcelle, C. 2002. FGFR4 signaling is a necessary step in limb muscle differentiation. Development 129: 4559-4569.

Relaix, F., Polimeni, M., Rocancourt, D., Ponzetto, C., Schafer, B.W., and Buckingham, M. 2003. The transcriptional activator PAX3-FKHR rescues the defects of Pax3 mutant mice but induces a myogenic gain-of-function phenotype with ligand-independent activation of Met signaling in vivo. Genes \& Dev. 17: 2950-2965. 
Relaix, F., Rocancourt, D., Mansouri, A., and Buckingham, M. 2004. Divergent functions of murine Pax3 and Pax7 in limb muscle development. Genes \& Dev. 18: 1088-1105.

Relaix, F., Rocancourt, D., Mansouri, A., and Buckingham, M. 2005. A Pax3/Pax7-dependent population of skeletal muscle progenitor cells. Nature 435: 948-953.

Shim, K., Minowada, G., Coling, D.E., and Martin, G.R. 2005. Sprouty2, a mouse deafness gene, regulates cell fate decisions in the auditory sensory epithelium by antagonizing FGF signaling. Dev. Cell 8: 553-564.

Stark, K.L., McMahon, J.A., and McMahon, A.P. 1991. FGFR-4, a new member of the fibroblast growth factor receptor family, expressed in the definitive endoderm and skeletal muscle lineages of the mouse. Development 113: 641-651.

Tajbakhsh, S. and Cossu, G. 1997. Establishing myogenic identity during somitogenesis. Curr. Opin. Genet. Dev. 7: 634641.

Taketomi, T., Yoshiga, D., Taniguchi, K., Kobayashi, T., Nonami, A., Kato, R., Sasaki, M., Sasaki, A., Ishibashi, H., Moriyama, M., et al. 2005. Loss of mammalian Sprouty2 leads to enteric neuronal hyperplasia and esophageal achalasia. Nat. Neurosci. 8: 855-857.

von Scheven, G., Alvares, L.E., Mootoosamy, R.C., and Dietrich, S. 2006. Neural tube derived signals and Fgf8 act antagonistically to specify eye versus mandibular arch muscles. Development 133: 2731-2745.

Weinstein, M., Xu, X., Ohyama, K., and Deng, C.X. 1998. FGFR-3 and FGFR-4 function cooperatively to direct alveogenesis in the murine lung. Development 125: 3615-3623.

Wiebe, P.O., Kormish, J.D., Roper, V.T., Fujitani, Y., Alston, N.I., Zaret, K.S., Wright, C.V., Stein, R.W., and Gannon, M. 2007. Ptfla binds to and activates area III, a highly conserved region of the Pdx1 promoter that mediates early pancreaswide Pdx1 expression. Mol. Cell. Biol. 27: 4093-4104.

Zhao, P. and Hoffman, E.P. 2004. Embryonic myogenesis pathways in muscle regeneration. Dev. Dyn. 229: 380-392.

Zhao, P., Caretti, G., Mitchell, S., McKeehan, W.L., Boskey, A.L., Pachman, L.M., Sartorelli, V., and Hoffman, E.P. 2006. Fgfr4 is required for effective muscle regeneration in vivo. Delineation of a MyoD-Tead2-Fgfr4 transcriptional pathway. J. Biol. Chem. 281: 429-438. 


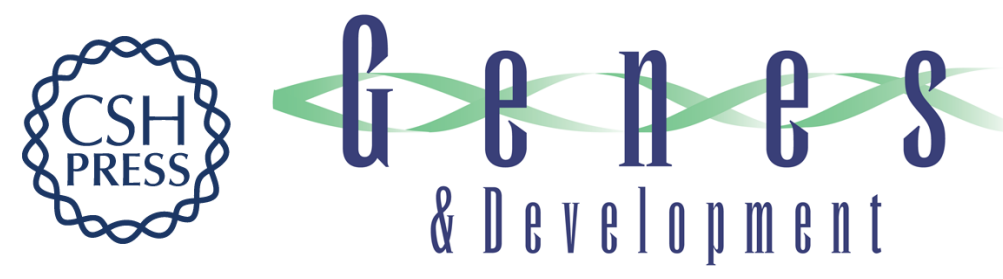

\section{Pax3 regulation of FGF signaling affects the progression of embryonic progenitor cells into the myogenic program}

Mounia Lagha, Jay D. Kormish, Didier Rocancourt, et al.

Genes Dev. 2008, 22:

Access the most recent version at doi:10.1101/gad.477908

Supplemental http://genesdev.cshlp.org/content/suppl/2008/07/01/22.13.1828.DC1
Material

References This article cites 36 articles, 17 of which can be accessed free at:

http://genesdev.cshlp.org/content/22/13/1828.full.html\#ref-list-1

License

Email Alerting

Receive free email alerts when new articles cite this article - sign up in the box at the top

Service

right corner of the article or click here.

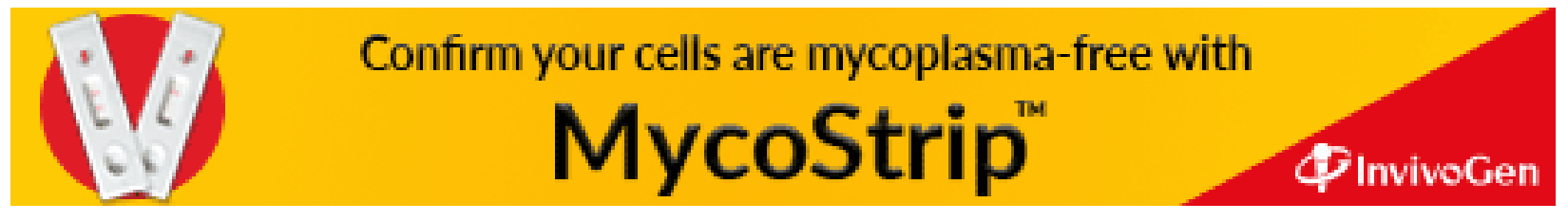

